\title{
Estrategias de inserción de inmigrantes caboverdeanos en los Estados Unidos, Portugal y en la Argentina
}

\author{
Marta M.Maffia*
}

En el presente trabajo me ocuparé de las estrategias que le han permitido a los caboverdeanos insertarse y construir una identidad social en la Argentina. Con el propósito de enriquecer la perspectiva de análisis haré referencia a los procesos migratorios y las modalidades de inserción adoptadas en los dos países en los que han migrado el mayor número de caboverdeanos: Estados Unidos y Portugal. Estas comparaciones de las cuales surgen, semejanzas y diferencias, darán sin dudas lugar a una mayor comprensión a los procesos gestados en la Argentina.

En el caso de la migraciones aludidas me he basado en la obra de autores de conocida trayectoria en la temática y complementariamente en observaciones realizadas durante breves períodos en ambos países. Mientras que, las consideraciones planteadas en relación al caso de Argentina, son el resultado de un exhaustivo trabajo de campo e investigación que incluyó el país de origen.

\section{Caboverdeanos en los Estados Unidos}

La conjunción de factores como las cíclicas sequías, el regimen de tenencia de la tierra, la política implementada por Portugal, entre otros factores, rompe sistemáticamente el precario equilibrio de la economía del archipiélago de Cabo Verde y es en ese equilibrio inestable, en el que se configura el fenómeno migratorio caboverdeano.

*Doctora investigadora del CONICET-Profesora titular UNLP-Argentina. 
En primer lugar me referiré a la situación de los inmigrantes de este origen en Estados Unidos, tomando básicamente como referencia la obra de Sydney Greenfield $(1976,1985,1990)$, Antonio Carreira $(1977 a, 1977 b, 1984)$ y Deirdre Meintel (1984).

Los caboverdeanos llegados a Estados Unidos ${ }^{1}$ lo hicieron como parte de la industria ballenera, de la cual New Bedford en el estado de Massachusets fue uno de los mayores centros, junto a Boston y Providence. La primer oleada llegó desde la isla de Brava, en esos primeros asentamientos mantuvieron muy poco contacto con otros segmentos de la población, y muy fuertes con sus parientes y amigos en su tierra natal. Cuando la industria de la pesca de la ballena declinó para la segunda mitad del siglo XIX, modificaron sus ocupaciones, se dirigieron a la industria textil y trabajos vinculados a las actividades portuarias. Para comienzos del siglo XX una flota de pequeñas y viejas embarcaciones a vela comerciaban y llevaban pasajeros entre New Bedford, Providence y Cabo Verde, manteniendo un constante contacto, no sólo con parientes y amigos de su tierra sino también con parientes asentados en otras partes del mundo, lo que podría ser pensado como un único universo socio-económico. Quienes participaban en él hablaban su lengua materna: el criol, identificándose e interactuando primariamente sólo con caboverdeanos.

El patrón general reportado por los informantes es el siguiente, un hombre joven deja su isla en compañía de parientes o amigos a menudo de su propia isla, a su arribo lo espera un pariente u otro caboverdeano, quien le encuentra trabajo en el mar o en tierra, donde otros coterráneos ya están trabajando. De esta manera se incorpora en una esfera familiar la que reduce el impacto al mínimo. Después de unos años él retorna, toma una esposa y constituye una familia, luego regresa a Estados Unidos y deja su familia en Cabo Verde. manda dinero a su esposa y parientes, a veces para comprar tierras y construir una casa. Usualmente hace periódicas visitas a la isla , retirándose allí cuando se jubila.

En ocasiones familias enteras se trasladan a Estados Unidos y forman parte de la comunidad caboverdeana del lugar.

Entre 1912 y 1943 un promedio de 466,4 personas dejan Cabo Verde anualmente para los Estados Unidos de acuerdo a fuentes oficiales. A ellos debemos sumar los caboverdeanos no registrados que se desplazaron en migraciones estacionales y en muchos casos como ilegales, a Cape Cod para el cultivo de los arándanos.

Estos trabajadores temporarios no provenían de la isla de Brava, fueron trabajadores pobres y generalmente sin ninguna instrucción reclutados en las islas de Santiago y Fogo. Aquí se hace necesario recordar lo expresado por Antonio Carreira (1977a:112): "la división de la sociedad insular en clases muy desequilibradas, en que la dominante trataba sobradoramente a las dominadas (en especial en Fogo) debe haber marcado mucho, desde el punto de vista psicológico, a los mulatos y a los más negrizados de los estratos más pobres".

Los nuevos inmigrantes caboverdeanos eran "más africanos" en apariencia 
que los primeros. Aunque erróneamente fueron llamados "bravas" o más peyorativamente "portugueses negros" y confundidos con los viejos inmigrantes de Brava, los nuevos no fueron aceptados en la comunidad caboverdeana ya establecida en New Bedford.

El gran control sobre la inmigración y las leyes restrictivas de 1921 y 1924 drásticamente redujeron el flujo de caboverdeanos hacia Estados Unidos. Luego la gran depresión, y la II guerra mundial y las aún más restrictivas leyes de 1950 pusieron fin al establecimiento de caboverdeanos en ese país.

Para las nuevas generaciones nacidas entre 1940 y 50 Cabo Verde no era más que un lugar distante y extraño de los que hablaban nostálgicamente sus padres y abuelos.

Según Greenfield (1976) la primer experiencia de qué significa ser negro en una sociedad blanca la tuvieron los jóvenes descendientes al ser incorporados al servicio militar, donde fueron ubicados en unidades segregadas y tratados no en forma diferente a otros negros, allí experimentaron el racismo.

Entre los caboverdeanos es usual encontrar representados ambos extremos del color, desde el altamente negroide a individuos blancos, aunque para el americano en general la mayoría de ellos son considerados mulatos.

Algunos nativos y sus descendientes han logrado éxito social y económico mientras que otros han fracasado para los estándares americanos. Algunos han obtenido una educación secundaria y hasta universitaria y otros nunca han completado la escuela primaria. Dada esta diversidad no es sorprendente que diferentes individuos tengan diferentes percepciones de la situación, que los conduzcan a decidir entre diversas alternativas en la búsqueda o construcción de su identidad (GREENFIELD, op.cit.).

En consecuencia, sostiene que los caboverdeanos adoptaron principalmente cuatro estrategias tendientes a lograr una mejor inserción en la sociedad de acogida. Una de ellas es la que denomina "caboverdeana-portuguesa", la segunda "caboverdeana-negra"; la tercera "caboverdeana-africana" y una cuarta la "caboverdeana-americana".

Con respecto a la primera. Después de la dura experiencia por la que pasaron los jóvenes descendientes de caboverdeanos, a raíz de la II Guerra muchos se definieron a sí mismos como portugueses. A esa definición se opusieron fuertemente azoreanos, madeerenses y portugueses continentales, temiendo que por implicación podrían ser asimilados con el grupo negro y degradados en la consideración social, ser discriminados y en consecuencia perder la oportunidad de obtener mejores posiciones en la estructura de la sociedad americana.

Está claro que el color fue el obstáculo principal que tuvieron que afrontar los caboverdeanos en Estados Unidos para construir una identidad social.

Los movimientos de derechos civiles desarrollados entre los 50 y los 60, para mejorar las oportunidades sociales, políticas y económicas de los americanos de color, los llevaron a la constitución, según Greenfield, de una estrategia de identidad que denominó: caboverdeana-negra. Los que sostienen esta posición 
rechazan su herencia caboverdeana, eligiendo definirse como negros americanos, trabajando para mejorar su posición en causa común con otros negros.

Muchos adoptan el estilo de ropa afroamericana, aprenden el inglés negro, dejan crecer su pelo a la moda de la comunidad negra y se casan con negros americanos, en un esfuerzo conciente de romper con la tradición que permitió a los caboverdeanos mantenerse como un grupo aparte.

Posteriormente los movimientos de independencia de África, entre ellos la de Cabo Verde y Guinea Bissau que se producen entre 1974-75, proveen una tercera estrategia identitaria, la caboverdeana africana. Los que son una minoría pueden verse a sí mismos como parte de un mundo mayoritario que está siendo explotado por una opresiva minoría blanca.

Esta posición tiene fuertes reacciones dentro de la propia comunidad caboverdeana ya que para gran parte de ellos las bases de su identidad están dadas como resultado de la mezcla entre europeos y africanos cuya consecuencia ha sido la constitución de una "etnía sui generis" que no es ni africana ni europea.

Finalmente, el relativamente reciente renacimiento y valorización de la etnicidad en Estados Unidos, ha conducido - según Greenfield - a la cuarta estrategia de proyección de la identidad étnica, la caboverdeana-americana. La táctica mayor es presentar a los caboverdeanos como una población única exitosamente adaptada a la vida en América.

Esta estrategia es un esfuerzo para ganar la aceptación de una identidad caboverdeana en términos étnicos, de manera que los miembros del grupo puedan competir en la sociedad más amplia libre del estigma asociado a los negros americanos.

En trabajos posteriores Greenfield (1985-90) señala que la estrategia caboverdeana-negra perdió sustento o soporte con la declinación de los Movimientos de Derechos civiles en los setenta.

Según este autor los caboverdeanos en Estados Unidos tienen que sobrellevar una carga más pesada que las de otras minorías, ya que ha sido un pueblo que ha sufrido los peores abusos, la esclavitud y el racismo, y aún hoy deben continuar enfrentando el racismo mientras tratan de hacerse un lugar para sí mismos y sus descendientes.

\section{Caboverdeanos en Portugal}

La composición social de los flujos migratorios en dirección a Portugal y con origen en Cabo Verde, no fue uniforme a lo largo de los tiempos. Antes de la Segunda Guerra Mundial emigraban las camadas socialmente más privilegiadas (comerciantes, propietarios, funcionarios públicos y estudiantes). La emigración cobra significación a partir de 1946 y especialmente en los años 60. En esta década, fue estimulada por el propio gobierno portugués, para responder a la falta de mano de obra provocada por la emigración portuguesa hacia Francia y la guerra colonial. La mano de obra caboverdeana fue canalizada, sobre todo, para 
el sector de la construcción civil (CARREIRA, 1977ạ; ARNALDO FRANÇA, 1992; SAINT MAURICE, 1994; LÓPEZ FILHO, 1980).

La lectura exploratoria de las estadísticas disponibles, por un lado, el conocimiento de la historia de la emigración caboverdeana y, simultáneamente de la emigración portuguesa, por otro, permitieron establecer tres factores / criterios diferenciadores asociados a la heterogeneidad de la población caboverdeana en Portugal: la naturalidad / nacionalidad, los períodos y las trayectorias migratorias ( SAINT MAURICE,1994).

Según el primero de los criterios se podrían determinar tres grupos: grupo I, constituido por individuos naturales (nativos) de Cabo Verde y con nacionalidad caboverdeana; grupo II, naturales de Cabo Verde pero con nacionalidad portuguesa; grupo III, natural de Portugal con nacionalidad caboverdeana.

Respecto al segundo criterio, los períodos de inmigración - con los cuales coinciden la mayor parte de los autores -, fueron: el primero, anterior a 1974; un segundo, entre 1974 y 1979 que se corresponde con el período de post-independencia de las colonias portuguesas y un tercero, que comenzó aproximadamente en los años 80 , con características diferentes a los anteriores: la inmigración de refugiados.

Finalmente, teniendo en cuenta los países de origen y residencia para la reconstitución de las trayectorias migratorias, se consideran como los más significativos: 1) Portugal; 2) Angola y Mozambique; 3) Cabo Verde, Santo Tomé y Guinea (estos dos últimos con poco peso) y 4) Otros países.

Por el cruzamiento de datos referidos a orígenes, períodos y trayectorias, Saint Maurice (1997) definió los siguientes grupos empíricos:

GRUPO I: caboverdeanos venidos directamente de Cabo Verde antes de 1974.

GRUPO II: caboverdeanos venidos de Santo Tomé.

GRUPO III: caboverdeanos venidos de otras colonias (Angola, Mozambique, Guinea) entre 1974 y 1979.

GRUPO IV: caboverdeanos venidos directamente de Cabo Verde después de 1974.

GRUPO V: caboverdeanos venidos de otros países.

Posteriormente clasifica los grupos según varios tipos de migraciones: migración laboral (GI, II y GV); migración política o de guerra (G III); perfil mixto (G IV).

De forma esquemática se podría decir que existen actualmente en Portugal dos situaciones polarizadas: una relativa a una inmigración esencialmente laboral, marcada por la presencia de una población donde predominan los sujetos en edad activa, una elevada tasa de actividad, de mano de obra no calificada (en la construcción civil y el sector servicios), elevados porcentajes de individuos analfabetos o con instrucción primaria, situación representada por los grupos 
con origen en Cabo Verde y Santo Tomé; la otra, está referida a una inmigración que caracteriza esencialmente una elite, con representación significativa entre los caboverdeanos que llegaron de Angola y Mozambique, fundamentalmente inmigración de refugiados, con instrucción media o superior y predominio de profesiones más calificadas.

Las áreas identificadas como problemáticas en la evaluación de integración inicial en la sociedad receptora, fueron por un lado, las condiciones habitacionales y la dificultad en el dominio de la lengua portuguesa destacadas por los entrevistados de bajo nivel cultural y poder económico. Respecto a la primera, la mayor parte de los emigrados vivían en lugares proporcionados por los empleadores, en casas de familiares o compatriotas, otros alquilaban pensiones (SAINT MAURICE,1994). Un número significativo viven en "barracas" (viviendas precarias), situación agravada por el hecho ser clandestinas y sin infraestructura sanitaria, viviendas situadas en "barrios degradados", "barrios de lata" o "barrios de barracas". En ellos se constituyen asociaciones que persiguen objetivos inmediatos y básicos en relación a las carencias de sus miembros.

La búsqueda de alojamiento en la proximidad de sus compatriotas, funciona, en la opinión de los entrevistados, como una defensa contra las agresiones exteriores.

En relación a la lengua, el portugués fue y aún es la lengua oficial del archipiélago y se adquiere casi con exclusividad en la escuela. Por lo tanto, en aquellos individuos emigrados con un bajo nivel de escolaridad, su manejo es deficiente, resultando en los niños una de las principales razones de fracaso escolar.

Respecto a las "interacciones", Saint Maurice (1994) señala: que los miembros del grupo de caboverdeanos que pasaron por Santo Tomé, y de los que vinieron más recientemente de Cabo Verde, establecen relaciones privilegiadamente con vecinos caboverdeanos; mientras que los de inmigración más antigua y venidos directamente de Cabo Verde se vinculan con vecinos portugueses. Dentro de ese último grupo, en el subgrupo de los que más recursos económicos y culturales poseen, la tendencia es a no cultivar siquiera las relaciones de vecindad con caboverdeanos, ya que residen en barrios con predominancia de portugueses, no hablar el "criol" en sus casas ni realizar en su vida diaria prácticas culturales típicamente caboverdeanas. Optaron por la nacionalidad portuguesa revelando una fuerte integración a la sociedad de acogida reforzada por la ausencia del deseo de regresar a Cabo Verde. A pesar de ello constituyen asociaciones insertas en las dinámicas urbanas que apuntan a objetivos culturales, políticos y de clase que no se cruzan con los de las asociaciones barriales (BORJA, 1998). Esta es una estrategia, que podríamos denominar en los términos de Greenfield, caboverdeana-portuguesa.

Las relaciones más claramente conflictivas son las que tienen los inmigrantes venidos directamente de Cabo Verde en períodos recientes (en muchos casos están en situación de "ilegalidad"), grupo que se ha revelado como el caso más ejemplar de "inserción segregada". En términos de interacción se limitan 
a su grupo (los caboverdeanos) habitando en barrios étnicos, manteniendo sus pautas culturales tradicionales y con poco contacto con los portugueses. Tienen nacionalidad caboverdeana, no pretenden adquirir la nacionalidad portuguesa y evalúan su inserción como negativa. No dudan de su regreso a Cabo Verde (SAINT MAURICE, 1994). Tal vez podríamos denominar a esta estrategia: caboverdeanacaboverdeana.

Para otros autores como el sociólogo Walter Rodrígues (1990) la inserción de la "minoría" caboverdeana en la sociedad portuguesa es definidamente marginal.

Finalmente un grupo muy particular de aquellos caboverdeanos que vinieron de otros países (fundamentalmente de Angola, Mozambique, Guinea) que se mantienen aislados del resto de la sociedad interactuando solamente con otros individuos provenientes de las ex colonias de habla portuguesa (PALOP), exceptuando los caboverdeanos. Esta podría ser, en términos de Greendfield, la estrategia caboverdeana-africana.

Por su lado, Arnaldo França (1992) considera la inserción de la comunidad caboverdeana en Portugal como "una extraña simbiosis de dos niveles", en un primer nivel la interacción entre caboverdeanos y portugueses transcurre de forma aceptable aunque surjan conflictos puntuales, generalmente en la esfera de las relaciones de menor proximidad. Pero en un nivel más abstracto, la interacción es sentida, mayoritariamente, como francamente negativa y se expresa a través de sentimientos que van de la inquietud a la agresión.

$Y$ "aunque raramente se reconozcan públicamente sentimientos racistas - sostiene el antropólogo caboverdeano Joao Lópes Filho (1980) - la población portuguesa en general posee esa mentalidad (muchas veces disfrazada)".

\section{Caboverdeanos en la Argentina}

La migración caboverdeana hacia la Argentina comienza a fines del siglo XIX con fecha muy imprecisa, cobrando relevancia a partir de la década de 1920, con la presencia de pequeños grupos o individuos provenientes de las islas de Sao Vicente, Santo Antao, en su mayoría, Sao Nicolau, Fogo y Brava, en menor medida. Otros períodos de mayor afluencia fueron entre 1927 a 1933 y el tercero después de 1946, decreciendo en intensidad alrededor de los años sesenta (MAFFIA, 1986). Período que coincide con el aumento del flujo migratorio de caboverdeanos hacia Portugal.

Los que migraron antes de la Independencia de Cabo Verde en 1975, lo hicieron con nacionalidad portuguesa, algunos de los cuales la mantuvieron hasta el final de sus días, la gran mayoría tramitó una nueva documentación caboverdeana (pasaporte) y son argentinos naturalizados.

En relación a las causas invocadas por los propios caboverdeanos coinciden en señalar que su migración fue impulsada fundamentalmente por razones laborales, en muy pocos casos se aducen razones políticas o de otra índole (MAFFIA,1986).

Respecto a las trayectorias migratorias, la mayor parte de los caboverdeanos 
que llegaron a la Argentina con pasaje pago (una de las modalidades), venían en barco directamente, con breve escala, en Dakar (Senegal), Lisboa (Portugal), Brasil, o Uruguay; mientras que los clandestinos "el destino" como ellos mismos expresan, determinaba la trayectoria y el final del viaje.

Datos estadísticos sobre esta población no aparecen ni en las Memorias de la Dirección Nacional de Migraciones ni en los censos, en primer lugar por la razón que entraron- los que lo hicieron legalmente- como portugueses, y segundo porque un gran número (difícil de determinar) entró clandestinamente. Tampoco ha habido investigaciones, hasta estos últimos años, sobre ese grupo, por lo tanto en los comienzos de mi trabajo en 1979, no tuve más alternativa que intentar censar a esta población, con el apoyo del en ese entonces cónsul honorario Joquim José Dos Santos, y miembros de la colectividad de Ensenada, de Dock Sud, La Plata y Capital Federal.

La zona que pudimos completar dada la sorprendente dispersión con la que encontramos en la Capital Federal y el gran Buenos Aires, fue La Plata, Berisso y Ensenada.

El censo realizado permite concluir para un universo de unas 260 unidades habitacionales y casi mil caboverdeanos entre nativos y descendientes, que la cifra más significativa de personas nativas de Cabo Verde comienzan a aparecer a partir de los 65 años predominando los hombres. Mientras que entre los descendientes de caboverdeanos hay un número mayor de mujeres que de hombres. Es de destacar que sólo el 6,8 \% de las parejas están constituidas por caboverdeanos ambos cónyuges, el resto tanto hombres como mujeres están unidos con personas de otro origen.

Respecto a la instrucción la mayoría absoluta de la población tiene instrucción primaria, completa el $50 \%$ y existe un relativamente buen porcentaje de personas que han accedido al nivel secundario, pero en su mayoría no lo completaron. El porcentaje de universitarios es bastante exiguo, sin embargo había una cifra significativa respecto a otros niveles de instrucción (cursos de inglés, música, pintura, modista, mecánica).

En relación a la ocupación: observamos que un $40 \%$ de la población ocupada, respecto de la que teóricamente podría estarlo, responde al parámetro para todo el país (para aquel período). La mayoría trabajando en relación de dependencia, un $85 \%$ de la población de ambos sexos, como obrero con y sin personal a cargo y como empleado con y sin personal a cargo. Solamente un $0,3 \%$ como patrón y un $14 \%$ por cuenta propia. Esto correspondería, comparándolo con un modelo de población socioceconómicamente hablando de medio para abajo.

Si realizamos el análisis por sexo, el resultado arroja que un $65 \%$ de los ocupados son hombres. Las ocupaciones más frecuentemente declaradas son maquinista, mecánico, cocinero (a bordo de barcos), policía, empleado de comercio, entre otras. En cuanto a las mujeres las mayores frecuencias señalan: empleada administrativa, modista y servicio doméstico.

Teniendo en cuenta los datos obtenidos respecto al nivel de escolarización, 
observamos un $50 \%$ de caboverdeanos que completaron el ciclo primario.

Respecto a la lengua materna (el "criol") es de notar que son muy pocas las personas menores de 30 años que la poseen, sólo a partir de esa edad comienza a aparecer definidamente, dándose con mayor frecuencia entre los 50-54 y los 70-74 años. En los hijos de estos inmigrantes, hay un gran predominio de lengua materna castellana (83\%) en todas las edades, no presentando dificultades en su escolarización.

Los informantes manifiestan, que la mayoría de los padres caboverdeanos no se preocuparon en enseñar el "criol" a sus hijos lo que nos permite inferir una mayor deseo de integración a la comunidad de adopción a través del dominio correcto del castellano. En general no han manifestado dificultades con respecto al aprendizaje del idioma castellano, el que de hecho hablan correctamente. Su adquisición fue rápida y sin grandes tropiezos.

Si nos referimos a los resultados de la muestra aleatoria sobre el anterior universo observamos, que disminuyeron como era de esperar los caboverdeanos nativos, ya que no ha habido inmigración en casi 40 años. Es llamativa la disminución de los varones descendientes de caboverdeanos nacidos en la Argentina, una razón podría ser que haya disminuido la fecundidad de esas personas y otra por las migraciones internas. Los de menos de 45 años tienen menor representatividad que el resto, lo que estaría indicando que posiblemente ese grupo de gente en edad productiva, posiblemente estaría migrando.

Respecto al origen combinado de los cónyuges se mantiene similar la proporción, aunque siempre hay levemente un mayor número de mujeres que eligen hombres de otro origen para casarse.

En relación a la ocupación, poco más de un tercio de los activos son varones mientras sólo la quinta parte de las mujeres están en esa condición.

Una décima parte del total trabajan por cuenta propia los restantes están en relación de dependencia como obreros o como empleados, en general sin personal a cargo. Si lo vinculamos con la escolarización, observamos que menos de la mitad tienen por debajo del ciclo básico y aproximadamente el $50 \%$ tienen el secundario completo.

La inserción en el mercado de trabajo es diferenciada, las principales ramas de actividad que los ocupan son comercio, industria y en el sector servicios, aunque los últimos registros y entrevistas señalan una tendencia de creciente desempleo y empobrecimiento de los inmigrantes y sus familias.

Con respecto a la inserción en la sociedad receptora en un primer momento, y tomando en consideración la intervención de redes informales, específicamente en relación a la búsqueda de habitación y empleo, y la posterior organización formal de esas redes para dar otros tipos de respuestas, podemos decir que: las redes informales en Argentina, se establecieron a lo largo de ejes o radios de circulación, los que constituyeron una vasta red de solidaridad con núcleos específicos (familias), en el interior de los cuales circulaban nuevos migrantes. Estos núcleos funcionando como verdaderos "microcontextos" originales, se encargaban 
de "albergarlos, buscarles trabajo, esposa, etc.", solidaridad que era más efectiva que aquella institucionalizada (por ejemplo, el Consulado de Portugal).

Estos microcontextos fueron los gérmenes de la "Sociedades" o "Asociaciones", es decir, los que con un regimen de autoridad y cumpliendo determinadas funciones, se constituyeron en instituciones (MAFFIA, 1986). Sin lugar a dudas como una situación en espejo del fenómeno de los emprendimientos asociativos-mutualistas de las grandes colectividades de inmigrantes radicadas en nuestro país.

Las primeras organizaciones caboverdeanas que se crearon, fueron las Sociedad de Socorros Mutuos de Ensenada en 1927 y la Unión Caboverdeana de Dock Sud (Avellaneda) en 1932, las que tenían por objetivo, cubrir, como ya dijimos, necesidades funcionales referidas a la ocupación, alojamiento, recreación y manifestaciones culturales de orden general. Algunos individuos proyectaron en esas organizaciones, sus propias necesidades de amparo, seguridad y situaciones de conflicto, que de alguna manera eran satisfechas y resueltas a través de las mismas.

Los lugares donde se establecieron esas asociaciones, podrían categorizarse tomando el concepto de la psicóloga social norteamericana Mónica Mc Goldrick (1982), como "barrios étnicos", espacios donde se restituyen algunos aspectos de la sociabilidad original y constituyen un punto de partida para insertarse en las diversas redes del proceso migratorio.

Los caboverdeanos se asentaron, como muchos otros inmigrantes (italianos, portugueses, griegos, polacos) en barrios del Dock Sud, la Boca y Ensenada en la provincia de Buenos Aires, fundamentalmente en relación, por un lado, a la cercanía con sus fuentes de trabajo, a bordo de los barcos de la marina mercante, no sólo argentina, sino de diversos países y de la Armada Nacional, y posteriormente a las fábricas, industrias y astilleros establecidos en la zona. Por otro lado, por la vecindad con parientes, amigos y coterráneos (si fuese posible de la misma isla).

A partir del censo y el muestreo pudimos detectar que más del $50 \%$ de la población en estudio, se habían desplazado a La Plata, Capital Federal y otros partidos del gran Buenos Aires, las razones aludidas fueron: el deseo de modificar su situación socioeconómica y lograr una mejor educación para sus hijos.

La generación de los viejos inmigrantes siguió viviendo en el barrio étnico, sus hijos y nietos nacidos en la Argentina, particularmente en la adolescencia, rechazan los valores tradicionales caboverdeanos y se "argentinizan", surgiendo en muchos casos conflictos intergeneracionales acompañados de profundos sentimientos de ambivalencia acerca de sus identificaciones étnicas.

Estos "viejos caboverdeanos" construyeron una imagen de sí mismos como portugueses, alejada de los "otros africanos", siguiendo el modelo construido por varias generaciones en Cabo Verde. Si bien trataron de adscribirse por lo menos nominalmente al segmento portugués de la población, de hecho fueron muy pocos los aceptados en sus ámbitos de sociabilidad.

En términos de las estrategias adaptativas de Greenfield, podría hablar de, en los inicios, una estrategia caboverdeana-portuguesa poco exitosa para la mayoría 
frente a la comunidad portuguesa local, ya que tampoco, como en Estados Unidos, los aceptaba en su seno. Y distinguimos una estrategia "caboverdeanaargentina". Esta estrategia con las diversas prácticas que la constituyen, llevó a la invisibilidad del grupo, posiblemente con el objetivo conciente o inconsciente, de lograr su inserción y reproducción social con el menor grado de conflicto posible. No es una elección conciente racional, es producto del sentido práctico como sentido del juego, adquirido históricamente. La misma, es producto de su experiencia histórica y de la dinámica establecida por el grupo con la sociedad de acogida, que desde las prácticas y el discurso han negado la presencia de negros en Argentina. Sin dejar de tener en cuenta otro aspecto, además, que muchos de ellos entraron clandestinamente quedando al margen (por un tiempo) de la estructura social, siendo de ese modo, invisibles para el Estado. Aprovechamos a establecer una relación con la marcada negativa de participación política² desde las Asociaciones, sobre todo de aquella participación que pudiese estar vinculada a ideologías que eran consideradas (por determinados grupos) "subversivas" del orden y que podría llevarlos a "visibilizarse" negativamente para el Estado y el resto de la sociedad.

He observado - no sólo en la generación de los inmigrantes, sino en sus descendientes ya sea que hablemos de una primera o segunda y hasta una tercera generación -, de una serie de prácticas (corporales, matrimoniales, de uso del espacio, rituales vinculadas al ciclo vital) y representaciones derivadas de esta estrategia, que Ilke B. Leite (1996:41) Ilama de "blanqueamiento-invisibilización". La misma posee sus raíces, como he señalado, en el pasado caboverdeano pero crece o supervive en contextos donde la invisibilidad se procesa por la producción de una cierta mirada, en la cual el negro es visto como no existente.

Según expone una de las informantes:

....el caso de negación fue el más exitoso de América Latina... todos los países apoyaron ese sistema de blanquear a la población, no hay ninguno que no lo haya hecho, pero acá el éxito fue arrasador, porque hasta los mismos negros no se dan cuenta que son negros....no lo admiten, es esquizofrenia porque no puede ser que un negro no se de cuenta, que no lo vea, que los negros sean otros...

$Y$ en ese proceso de negación algunos caboverdeanos reconocen y califican la acción de la colonización portuguesa como:

Fue devastadora...que nosotros somos diferentes, que somos más inteligentes, que somos más lindos, más cultos, toda esa historia todos los caboverdeanos hasta el más inculto se la creen y la repiten, que no tienen nada que ver con los africanos del continente.

Recordando la relación que B. Leite (op.cit) establece entre el mecanismo de la invisibilización y el racismo, considero que como parte de ese mismo proceso, se da la negación o la no conciencia del mismo. Hoy, este proceso está 
comenzando a ser revertido por pequeños grupos de jóvenes de segunda y tercera generación, que junto a otras minorías de afrodescendientes, exacerban la crítica a la exclusión y a la invisibilidad, reivindicando sus orígenes y afiliaciones diaspóricas ${ }^{3}$.

\section{Algunas consideraciones finales}

Las diferencias socioculturales, económicas y fundamentalmente las étnicoraciales, parecen estar en la base de la explicación de las diferentes estrategias de inserción de los caboverdeanos en los tres países. La "etnía" marca con fuerza a esta población, orientando las acciones y relaciones que se establecen con los países de destino. Tal como sostiene la socióloga Maria Beatriz Rocha Trindade (1995), cuando las diferencias étnicas son dominadas por diferencias raciales, tales divisiones se tornan más acentuadas, obligándolos en muchos casos a desarrollar mecanismos de defensa, entre ellos el recrear en la medida de lo posible su lugar de origen (hasta el caso extremo de constituirse en guetos) y a partir de allí negociar su inserción en la sociedad .

Los caboverdeanos que emigraron y se establecieron en la Argentina - tema de análisis de este trabajo - adoptaron por largos períodos estrategias (con matices diversos) que condujeron a la invisibilidad del grupo, "dilución" que les permitió una inserción y reproducción social con bajo nivel de conflicto aunque con poca movilidad social ascendente. Hoy en otro contexto sociohistórico su presencia se torna expresiva (individual y grupalmente), nuevos espacios están siendo conquistados particularmente por sus descendientes, ya sea en términos de movilidad social, de expresión cultural, académica y de participación política.

Acciones que nos conducen a preguntarnos, si esta búsqueda de visibilidad junto a otras minorías de afrodescendientes de la Argentina, podemos considerarla, como parte de una nueva estrategia que está en marcha, a la que podríamos llamar "afrodiaspórica", centrada en la lucha contra la discriminación y el racismo y por el reconocimiento pleno de sus derechos ciudadanos. Debemos seguir investigando en ese sentido.

\section{Notas}

1 - Antonio Carreira señala que, a pesar de relativa confianza que le merecen las estadísticas de esa época, la llegada de los primeros caboverdeanos a los Estados Unidos podría situarse entre los años 1685 y 1700 .

2 - En 1910, se sanciona la Ley de Defensa Social, que reglamenta la admisión de extranjeros en el territorio argentino, legitimando la expulsión y restricción de su ingreso, identificando explícitamente aquellas ideologías como el anarquismo y el socialismo, que pudiesen atentar contra la seguridad nacional. Ello motivó más de 2000 enviados a Ushuaia y la deportación de un buen número de extranjeros, que en su mayor parte recala en Uruguay. La xenofobia, hará en adelante una especie de contrapunto con el cosmopolitismo inherente a la sociedad culta y liberal, abierta a la proveniencia europea (CLEMENTI, 1984: 74-75). 
3 - Para los descendientes de inmigrados y los pueblos en diáspora, el territorio de origen constituye un recurso siempre disponible, asimismo cuando las semejanzas culturales y lingüísticas ya se apagaron (GANS, 1979).

\section{Referencias}

BOAVENTURA LEITE, I. (Org.). Negros no sul do Brasil. Santa Catarina: Letras Contemporâneas, 1996.

BORJA, Orlando. A segunda geração de imigrantes cabo-verdianos em Portugal: processos de inserção social. In: Cultura. Cabo Verde, № 2, Ministério da Cultura, Praia, Cabo Verde, 1998.

CARREIRA, António. Migrações nas ilhas de Cabo verde. Lisboa: Universidade Nova de Lisboa, 1977a.

CARREIRA, António. Cabo Verde. Clases sociales, estructura familiar, migraciones. Lisboa: Biblioteca Ulmeiro, 1977 b.

CARREIRA, António. Cabo Verde (aspectos sociais, seca e fomes do século XX. Lisboa: Ulmeiro, 1984.

FRANÇA, Arnaldo. Imigrantes caboverdeanos em Portugal. Lisboa: Instituto de Estudos para o desenvolvimento, 1992.

CLEMENTI, Hebe. El miedo a la inmigración. Buenos Aires: Leviatán, 1984.

GANS, Herbert. Symbolic ethnicity: the future of ethnic groups and cultures in America. In: Ethnic and Racial Studies. Vol. II. 1, 1979, p. 1-20.

GREENFIELD, Sidney. In search of the Social Identity: Strategies of ethnic Identity. Management among Capeverdians in Southeastern Massachussetts. In: Luzo-Brazilian Review, Vol.13, №1, 1976.

GREENFIELD, Sidney. Barbadian in the Amazon and Cape Verdeans in New England: contrast in adaptations and relations with Homelands. In: Racial Studies,Vol. 8, № 2, 1985.

GREENFIELD, Sidney. The Cape Verde Islands: Their settlement, the emergence of their creole culture, and subsequent migrations of their people. In: Portuguese Migration in Global Perspective. Toronto: Ed. David H. Higgs. The Multicultural History Society of Ontario, 1990.

LÓPES FILHO, João. O emigrante cabo-verdiano em Lisboa. In: Africa Literatura-Arte-Cultura. № 9, 1980, p. 12.

MC GOLDRICK, Mónica. Ethnicity and Family. An overview. En Mc Goldrick, Pearce and Giordano (comps.) Ethnicity and Family Therapy. New York: Guilford Press, 1982, p. 3-29.

MAFFIA, Marta. La inmigración caboverdeana hacia la Argentina. Análisis de una alternativa. In: Trabalhos de Antropología e Etnología. Vol. 25, Porto: Sociedade Portuguesa de Antropología e Etnología, 1986.

MEINTEL, Deirdre. Emigração em Cabo Verde: solução ou problema? In: Revista Internacional de Estudos Africanos. № 2, junho-dezembro, 1984, p. 93-120.

ROCHA-TRINDADE, M. B. Sociologia das Migrações. Lisboa: Universidade Aberta, 1995.

RODRÍGUES, Walter. Comunidade caboverdiana: marginalização e identidade. In: Revista 
Estudos. ISCTE, Lisboa, 1990, p. 96-103.

SAINT MAURICE, Ana. Reconstrução das identidades no processo de emigração. A população caboverdiana residente em Portugal. Dissertação de doutoramento em Sociologia pelo Instituto Superior de Ciências do Trabalho e da Empresa. Portugal, 1994.

\title{
RESUMEN
}

El presente trabajo trata sobre las estrategias que le han permitido a los inmigrantes caboverdeanos insertarse y construir una identidad social en la Argentina. Con el propósito de enriquecer la perspectiva de análisis hace referencia a los procesos migratorios y las modalidades de inserción adoptadas en los dos países en los que han migrado el mayor número de caboverdeanos: Estados Unidos y Portugal. Estas comparaciones de las cuales surgen, semejanzas y diferencias, darán sin lugar a una mayor comprensión a los procesos gestados en la Argentina.

Palabras claves: migración caboverdeana; inserción; Argentina.

\begin{abstract}
This paper discusses the strategies that have allowed Cape Verdeans immigrants in Argentina to settle and build a social identity. In order to enrich the perspectives of analysis, migratory processes and integration patterns in United States and Portugal, the two main countries to which most Cape Verdeans have migrated are considered. Similarities and differences arisen from these comparisons will lead to a greater understanding of the processes that are taken place in Argentina.
\end{abstract}

Keywords: Cape Verdean migration; integration; Argentina. 